\title{
Moving Filter Applied to Axial Transverse Tomography
}

\author{
Tadayoshi Matsuda \\ Department of Radiology, Toyohashi City Hospital, Toyohashi
}

\begin{abstract}
An application of the moving filter attached to the radiation mouth of the axial transverse tomograph reduces exclusively the $\mathbf{x}$-ray irradiation dose to the contour area of the cross section of the body.

By means of this filter the density of the axial transverse tomogram is equalized from the periphery to the center of the cross section, thus making possible a wide application of the axial transverse tomogram to the radiation therapy.
\end{abstract}

In the axial transverse tomogram there is a tendency that the radiation density becomes greater in the soft tissues of the body surface than in the internal structures, with resulting difficulty in an evaluation of the cross sectional contours. To remove this defect $x$-rays projected on the contour of a subject in rotation radiography should be made smaller than those projected on the center of the body. For such a purpose a moving filter is devised and employed.

Moving filter and its structure

A moving filter is attached to the front of the radiation mouth of the axial transverse tomograph of the horizontal type (Fig. 1). The main part consists of a thin metallic diaphragm, leaf $A$, which is designed to be tapered at its free end and to widen or narrow the radiation opening in a direction parallel with the rotating axis of the apparatus. The diaphragm acts also as a partial filter owing to its thinness. The contour of the transverse section of the body is seldom circular and usually oval or irregular, so that leaf $\mathrm{A}$ is preset by means of the handle $\mathrm{D}$ and light projector $\mathrm{L}$ to widen or narrow in conformity with the above shapes, and at operation the leaf A is made to move by means of a small motor B (Fig. 2).

When the radiography room is kept dark and lamp $\mathrm{L}$ lighted, the light rays through the diaphragm illuminate the contour of the patient lying unclothed on the radiography table. By next placing the $x$-ray tube at various angles, $\phi_{1}, \phi_{2}$ or $\phi_{0}$, it is possible to know how large the size of the irradiation aperture should be. If now the $\mathrm{x}$-ray tube is rotated through angle $\phi_{1}$ in a horizontal plane from $f_{1}$ to $f_{2}$, the tapered edge of the leaf $W_{1}$ will shield a peripheral part of the body so that $\mathrm{x}$-rays entering this part will be reduced (Fig. 3). As the $\mathrm{x}$-ray tube moves from $\phi_{2}$ to $\phi_{3}$, the leaves move synchronously with the gear rotating the $\mathrm{x}$-ray tube and widens or narrows the radiation opening. 


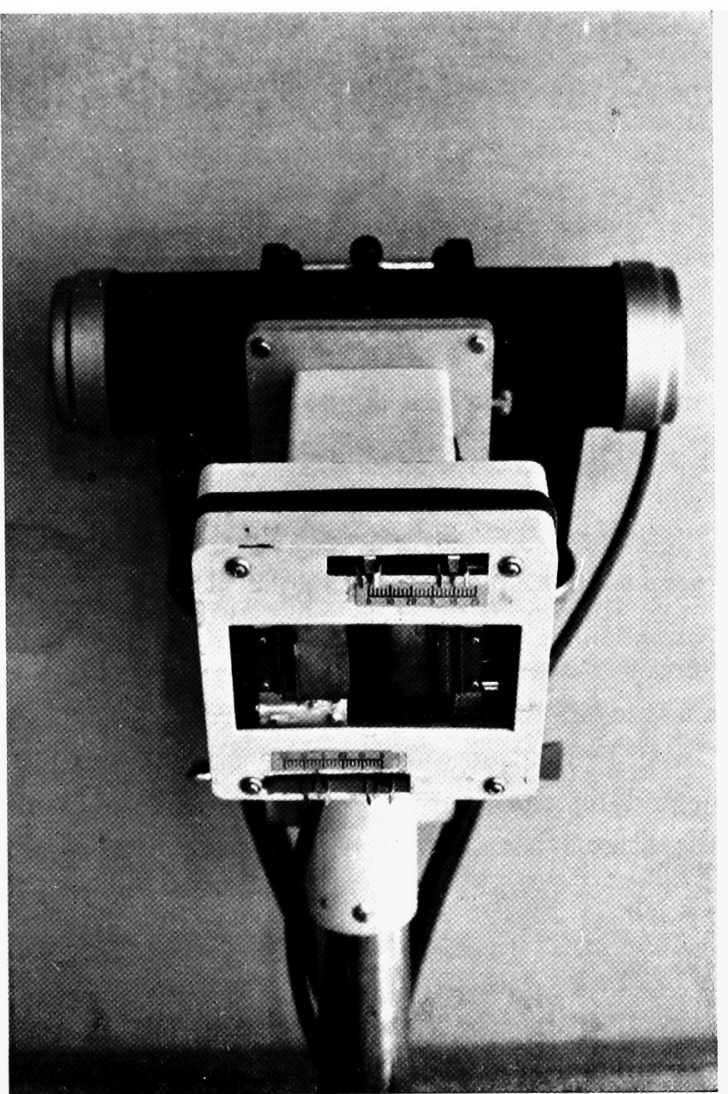

Fig. 1

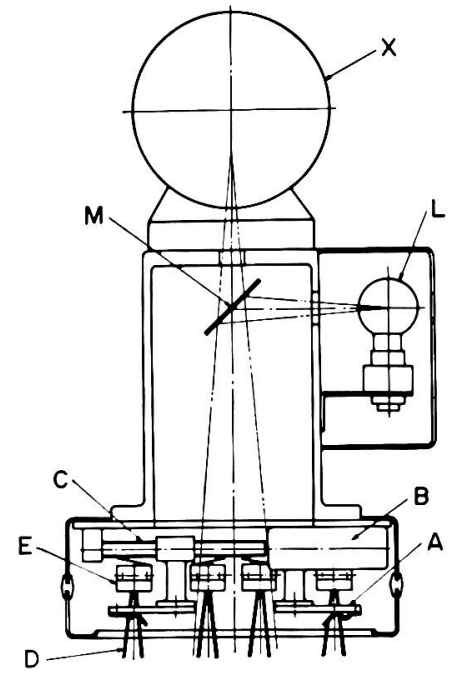

Fig. 2

Fig. 1. Moving filter attached to the radiation mouth.

Fig. 2. Construction of the moving filter.

A : leaf of filter. B: motor. C: screw. D: handle. L: light projector. M: mirror. $\mathrm{X}$ : x-ray tube.

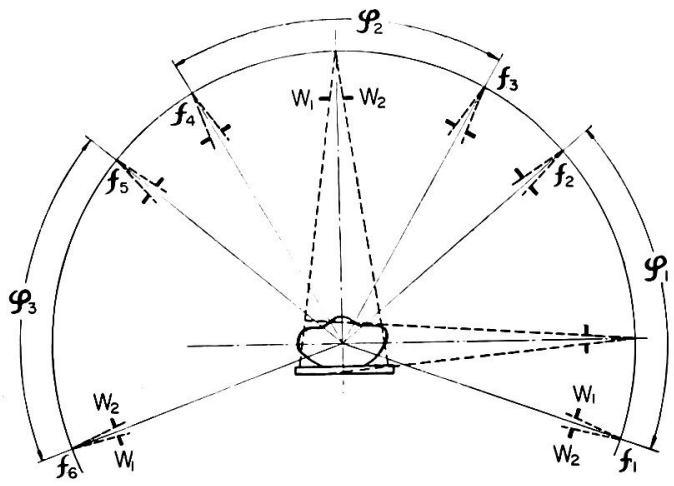

Fig. 3. Mechanism of work of moving filter.

Leaves $W_{1}$ and $W_{2}$ absorb adequately $x$-ray irradiating the contour of the cross section of the body in the range of rotation from $f_{1}$ to $f_{2}$. 


\section{Advantages in the use of the moving filter}

In the axial transverse tomography $\mathrm{x}$-ray after passing the subject will differ in dose according to the peripheral or central parts of the body on account of differences in its penetration distance. A description will now be made of a case of neck tomography. At the level of the larynx, the thickness of the neck was measured for every $10^{\circ}$ at points corresponding to depths of $2 \mathrm{~cm}, 4 \mathrm{~cm}$ and the center of the body from the skin surface (Fig. 4, A). When the results are plotted, the rotation angle being the abscissa and the thickness the ordinate, a curve like $B$ is obtained by connecting the marks (Fig. $4, \mathrm{~B}$ ). Of the 4 curves $\mathrm{C}$ and $\mathrm{D}$ are nearly parallel to the ordinate but $A$ and $B$ show small values of thickness in the ranges of $0^{\circ}-60^{\circ}$ and $160^{\circ}-220^{\circ}$ as the distance of $\mathrm{x}$-ray penetration is increased. The $x$-ray dose that influences the density of these various points of the transverse sec-

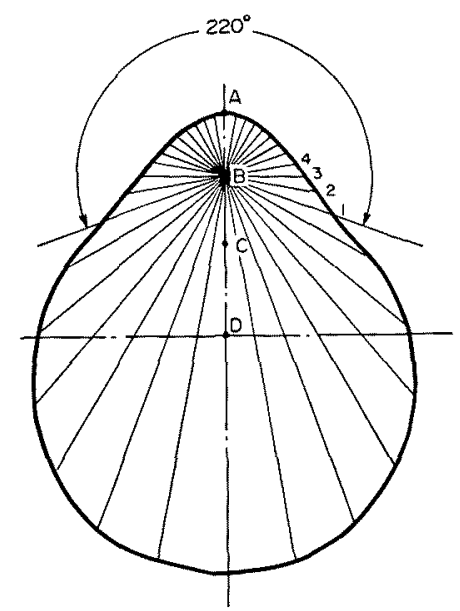

Fig. 4, A. Points A, B, C and D are arranged on the sagittal line with distances of $0,2,4$ and $6 \mathrm{~cm}$ from the skin surface. Angle $220^{\circ}$ shows the range of rotation.

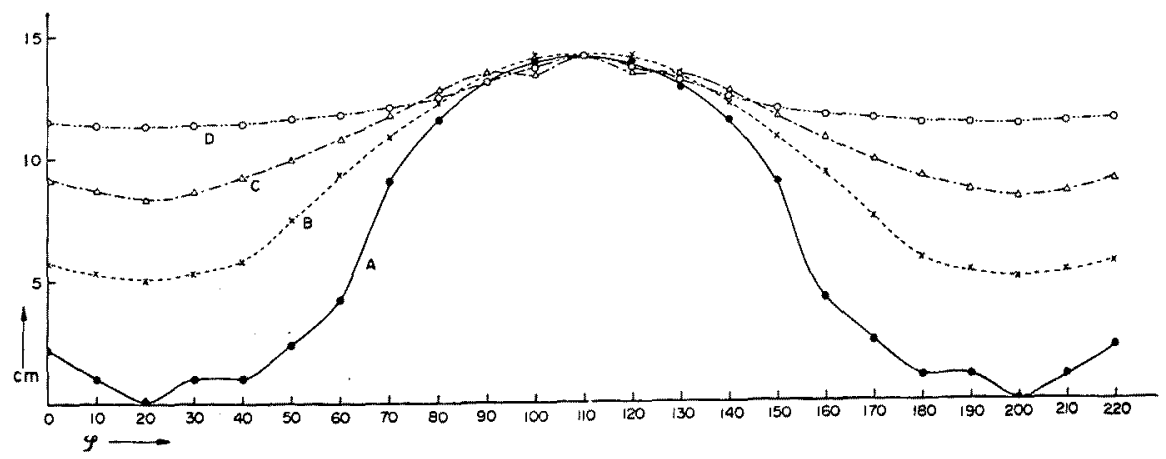

Fig. 4, B. Relation of diameters of the neck measured at points A, B, C and D (ordinate) and rotation angle (abscissa). 
tion surface is based on the integration of x-ray doses remaining after passing through these varying thicknesses. Hence, in order to equalize the densities of points $\mathrm{A}, \mathrm{B}$ and $\mathrm{C}$ with that of $\mathrm{D}$, it is necessary to avoid too great a difference in thickness and to compensate the difference by the use of an adequate filter. The same holds for other parts of the body.

\section{Selection of the thickness of the moving filter}

A phantom of various parts of the body made of Mix D was used. The radiographic conditions of the axial transverse tomography with $65 \mathrm{kVp}, 20 \mathrm{~mA}$, $12 \mathrm{sec}$, the distance of $\mathrm{x}$-ray tube from the film being $230 \mathrm{~cm}$, gave the following results: When a moving filter was not used, the density of the tomogram that imaged the front of the neck $2.5 \mathrm{~cm}$ in depth from the surface did not differ much from that of the ground density of the film, and the distinction of the actual figure of the neck was impossible (Fig. 5, A).

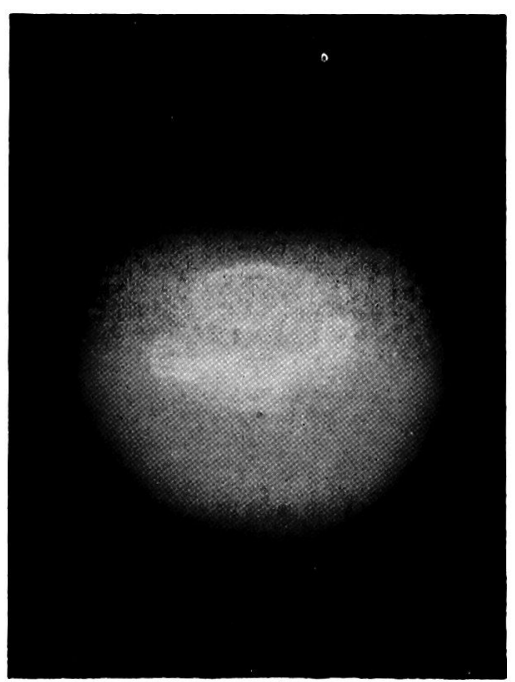

A

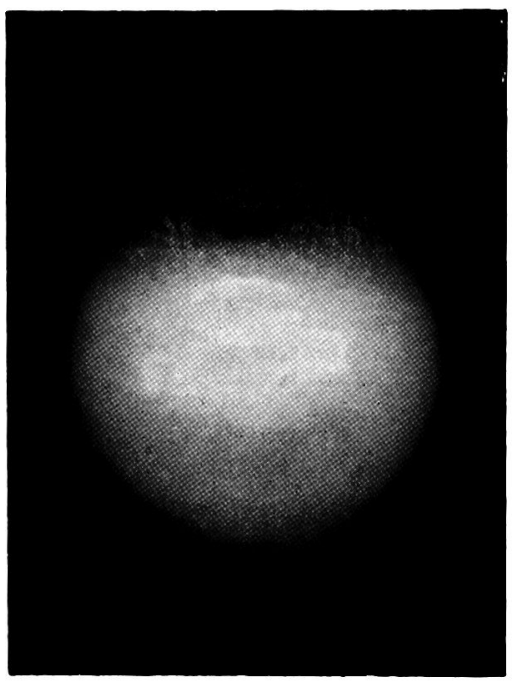

B

Fig. 5. Axial transverse tomogram of the neck.

A: Taken without moving filter. The contour of the laryngeal prominence is not inspected.

B: Taken by using moving filter. The contour of the laryngeal prominence is imaged.

In order to equalize $x$-ray density at the superficial part of the neck and at the central part of the axial transverse tomogram, it was found necessary to use a tapered filter of ground thickness of $3 \mathrm{~mm} \mathrm{Al}$ with an additional $\mathrm{Cu}$ leaf of $0.2-0.3$ $\mathrm{mm}$ shielding $3 \mathrm{~cm}$ of the peripheral part of the neck during the rotation of the $\mathrm{x}$-ray tube through the range of $0^{\circ}-50^{\circ}$ and $170^{\circ}-220^{\circ}$ (Fig. 5, B).

In order to reduce the $\mathrm{x}$-ray dose to fit the body contour there is a need to adjust the tube voltage as well as the thickness of the moving filter. After examination on 160 adults the exposure conditions of a moving filter suited for 
TABLE 1. Shield of body surface with moving filter

\begin{tabular}{l|r|r|r|r|r|r}
\hline & \multicolumn{2}{c}{ Exposure condition } & \multicolumn{2}{c}{ Moving filter } & \multicolumn{2}{c}{ Body shield of } \\
& $\mathrm{kVp}$ & $\mathrm{mA}$ & $\mathrm{Al}$ & $\mathrm{Cu}$ & $\begin{array}{c}\text { Anterior } \\
\text { part }\end{array}$ & $\begin{array}{c}\text { Lateral } \\
\text { part }\end{array}$ \\
\hline Head & 110 & 5 & $4 \mathrm{~mm}$ & $0.4 \mathrm{~mm}$ & $3 \mathrm{~cm}$ & $2 \mathrm{~cm}$ \\
Neck & 95 & 5 & $4 \mathrm{~mm}$ & $0.3 \mathrm{~mm}$ & $3 \mathrm{~cm}$ & \\
Chest & 120 & 3 & $4 \mathrm{~mm}$ & $0.5 \mathrm{~mm}$ & $3 \mathrm{~cm}$ & $3 \mathrm{~cm}$ \\
Pelvis & 115 & 10 & $4 \mathrm{~mm}$ & $0.7 \mathrm{~mm}$ & $3 \mathrm{~cm}$ & $3 \mathrm{~cm}$
\end{tabular}

axial transverse tomograms of various body areas were found to be as shown in Table 1, as regards thickness of the filter, its distance from body surface for effective shielding, and range of rotation.

\section{Comment}

Even with conventional normal radiography, for example, the spinal column in its whole length is radiographed on a single film, when a filter device is used in order to make the $\mathrm{x}$-ray dose at the thoracic and lumbar spines approximately equal on the film. ${ }^{1}$ In order to produce the contours of an axial transverse tomogram as sharply as possible the following methods have hitherto been employed. One is to depict the contours by inspecting with light brighter than in the usual viewing box, and the other to paint contrast medium on the body surface or attach lead threads and conduct tomography. ${ }^{2}$ To these methods the use of a moving filter is superior, since an approximately homogeneous density can be obtained by a single procedure. The principle of moving the filter during tomography is based on the principles of the mechanism of moving the diaphragm in conformation radiotherapy. ${ }^{3}$ To make the most of the special advantages of the axial transverse tomography it is necessary to have an exact information about the internal conditions as well as the contours as a whole. This method has come into use more and more frequently for planning the radiotherapy with increasing need to depict the contours of the transverse section as accurately as possible.

\section{References}

1) Ediger A., Gajewski, H. \& Gepp, H. Röntgen-Ganzaufnahme der Wirbelsäule. Fortschr. Röntgenstr., 1956, 84, 365-371.

2) Matsuda, T. \& Watanabe, T. On the role of axial transverse tomography during rotation convergent irradiation. Nippon Acta radiol., 1959, 18, 1584-1591.

3) Takahashi, S. Conformation radiotherapy. Rotation techniques as applied to radiography and radiotherapy of cancer (Acta radiol suppl. 242). 1. ed., Acta radiol, Stockholm, 1965, 30 . 